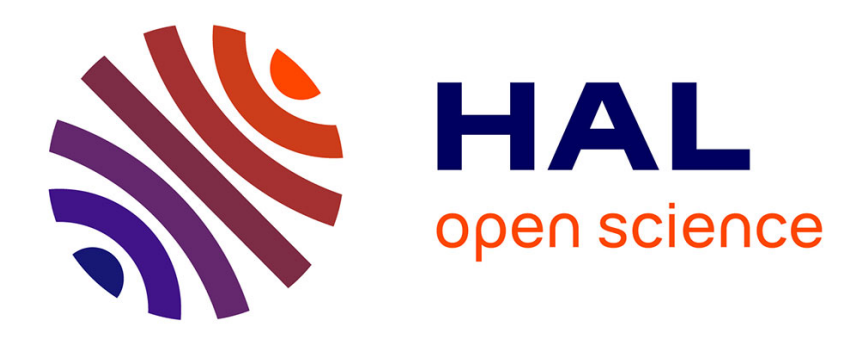

\title{
Cardinal extensions of EU model based on the Choquet integral
}

\author{
Alain Chateauneuf, Michèle Cohen
}

\section{To cite this version:}

Alain Chateauneuf, Michèle Cohen. Cardinal extensions of EU model based on the Choquet integral. 2008. halshs-00348822

\section{HAL Id: halshs-00348822 \\ https://shs.hal.science/halshs-00348822}

Submitted on 22 Dec 2008

HAL is a multi-disciplinary open access archive for the deposit and dissemination of scientific research documents, whether they are published or not. The documents may come from teaching and research institutions in France or abroad, or from public or private research centers.
L'archive ouverte pluridisciplinaire HAL, est destinée au dépôt et à la diffusion de documents scientifiques de niveau recherche, publiés ou non, émanant des établissements d'enseignement et de recherche français ou étrangers, des laboratoires publics ou privés. 


\section{Documents de Travail du Centre d'Economie de la Sorbonne}

C

E

$\mathrm{S}$

W

0

1

k

方

$\mathrm{P}$

a

$\mathrm{p}$

e

$r$

$\mathrm{S}$

CENTRE NATIONAL

DE LA RECHERCHE

SCIENTIFIQUE

Maison des Sciences Économiques, 106-112 boulevard de L'Hôpital, 75647 Paris Cedex 13

http://ces.univ-paris1.fr/cesdp/CES-docs.htm

ISSN : 1955-611X the Choquet integral

2008.87 


\title{
Cardinal extensions of EU model based on the Choquet integral
}

\author{
Alain Chateauneuf and Michèle Cohen \\ Paris School of Economics and \\ CES-University Paris I Panthéon-Sorbonne \\ 3rd December 2008
}




\section{Résumé}

Ce chapitre d'ouvrage collectif vise à présenter les extensions du modèle EU, basées sur l'intégrale de Choquet, qui permettent de tenir compte des comportements observés comme dans le paradoxe d'Allais dans le risque ou le paradoxe d'Ellsberg dans l'incertain, où il y a violation du modèle d'espérance d'utilité. Sous l'axiome clé d'indépendance comonotone, Schmeidler dans l'incertain, et Quiggin et Yaari dans le rique ont réussi à caractériser des préférences qui généralisent le modèle EU, à l'aide d'une fonctionnelle qui s'avère être une intégrale de Choquet. Ces modèles expliquent la plupart des paradoxes observés et permettent des comportements plus diversifiés à la fois dans l'incertain et dans le risque.

Mots clé: Incertain, Risque, Comonotonie, Capacité de Choquet, Intégrale de Choquet.

JEL: D81

\section{Abstract}

This chapter of a collective book aims at presenting cardinal extensions of the EU model, based on the Choquet integral, which allow to take into account observed behaviors as in Allais' paradox under risk or Ellsberg's paradox under uncertainty, where the expected utility model is violated.

Under a key axiom, the comonotonic independence axiom, Schmeidler under uncertainty, and Quiggin and Yaari under risk, succeeded to characterize preferences which generalize the EU model, by means of a functional that turned out to be a Choquet integral. These models not only explain most of the observed paradoxes but also allow for more diversified patterns of behavior under uncertainty as well under risk.

Key words: Uncertainty, Risk, Comonotony, Choquet capacity, Choquet integral.

JEL: D81 


\section{Introduction}

The classical model of decision under risk, the Expected Utility model (von Neumann and Morgenstern (1947)), and under uncertainty, the Subjective Expected Utility model (Savage, (1954)) have been proved to be often violated by observed behaviors, the most famous evidence being Allais paradox under risk and the Ellsberg paradox under uncertainty.

Among others, these two paradoxes have called into question these classical models.

To take into account these behaviors, Schmeidler $(1982,1989)$ under uncertainty, and Quiggin (1982) and Yaari(1987) under risk have built new axiomatizations of behavior for which the EU (or SEU) model is a particular case.

Under a key axiom, namely the comonotonic independence axiom, an appealing and intuitive axiom requiring that the usual independence axiom holds only when hedging effects are absent, Schmeidler, Quiggin and Yaari have, independently, succeeded to characterize the preferences by means of a functional that turned out to be a Choquet integral, under uncertainty as well as under risk. Choquet integral thus proved to be an important tool for decision making under risk and uncertainty.

Moreover, not only these models - with the generic term of Choquet Expected Utility (CEU) - explain most of the observed paradoxes but they also offer simple but flexible representations, allow for more diversified patterns of behavior under uncertainty as well as under risk, and especially allow to separate perception of uncertainty or of risk from the valuation of outcomes.

The aim of this paper is mainly to emphasize the role of the models of behavior based on Choquet integral.

\subsection{Notations and definitions}

Let us recall some notations of the previous chapters.

$S$ is the set of states of nature, $\mathcal{E} \subset 2^{S}$ a sigma-algebra of subsets of $S$, $A \in \mathcal{E}$ an event and $\mathcal{C}$ the set of consequences. A decision is identified to an act, which is a mapping from $S$ to $\mathcal{C}$.

The set of acts will be denoted $\mathbb{V}$ when the set of consequences is a set $\mathcal{C}$.

The preferences between acts are represented by a binary relation denoted $\succsim$ on the set of acts $\mathbb{V}$. Strict preference will be denoted $\succ$ and indifference $\sim$. The preference relation on $\mathbb{V}$ induces (thanks to constant acts) a preference relation on the set $\mathcal{C}$ of consequences. Abusing notation, we equally denote $\succsim$ this preference relation on $\mathcal{C}$.

An act $f$ is a simple step act if there exists a finite partition $\left\{A_{i}, i \in I\right\}$ of $S$, with $A_{i} \in \mathcal{E}$ for all $i \in I$, such that $f\left(A_{i}\right)=\left\{c_{i}\right\}$ - a singleton. When $f$ is a simple step act, we will denote $f=\left(c_{1}, A_{1} ; \ldots ; c_{n}, A_{n}\right)$ where $c_{1} \precsim \ldots \precsim c_{n}$. In particular, a constant act, $\delta_{c}$ is characterized by $\delta_{c}(S)=\{c\}, c \in \mathcal{C}$. 
In the sequel we will need to differentiate the two following particular cases:

- When the set of consequences is a subset of $\mathbb{R}$ and consequently already ordered, the set of acts will be denoted $\mathbb{X}$. Furthermore, a simple step act will be denoted $X=\left(x_{1}, A_{1} ; \ldots ; x_{n}, A_{n}\right)$ where $x_{1} \leq \ldots \leq x_{n}$

Defining the characteristic function of $A_{i}: 1_{A_{i}}$ by : $1_{A_{i}}(s)=1$ if $s \in$ $A_{i}, 1_{A_{i}}(s)=0$ otherwise, one denotes also by abusing notation, $X=$ $\sum_{i=1}^{i=n} x_{i} 1_{A_{i}}$.

- When the set of consequences $\mathcal{Y}$ is a set of lotteries (or equally, distributions of probability with finite support) on a given set $\mathcal{C}$ of outcomes and when the set $S$ of states of nature is finite, the set of acts, equally the set of mappings from $S$ to $\mathcal{Y}$, will be denoted $\mathbb{F}_{0}$. In this case acts are called "horse lotteries". The set of consequences $\mathcal{Y}$ being a mixture set (see chapter 1), one can use this structure in order to define, for all $f$ and $h$ in $\mathbb{F}_{0}$ and all $\alpha$ de $[0,1]$, the act $\alpha f+(1-\alpha) h$ by :

$(\alpha f+(1-\alpha) h)(s)=\alpha f(s)+(1-\alpha) h(s)$ for all $s$ in $S$.

For this operation, $\mathbb{F}_{0}$ is also a mixture space.

\subsubsection{The notion of comonotony.}

The notion of comonotony appear to be crucial for the axiomatic of models that we will develop in this chapter.

Definition 1 Two acts $f$ and $g$ of $\mathbb{V}$ are said to be comonotonic ${ }^{1}$ if there exists no pair $s, s^{\prime} \in S$ such that $f(s) \succ f\left(s^{\prime}\right)$ and $g(s) \prec g\left(s^{\prime}\right)$.

When the set of consequences is a subset of $\mathbb{R}$ ordered with the usual order $\geq$, the definition turns out to be:

Definition 2 Two acts $X$ et $Y$ of $\mathbb{X}$ are said to be comonotonic if for all $s, s^{\prime} \in S$,

$$
\left(X(s)-X\left(s^{\prime}\right)\right)\left(Y(s)-Y\left(s^{\prime}\right)\right) \geq 0
$$

If two acts $X$ and $Y$ are comonotonic, they both "yield" at least the same payment in state $s$ than in state $s^{\prime}$ and consequently any positive linear combination of these two acts will preserve this order. Thus, it is impossible to insure against the "variability" of the payments of a financial asset $X$, by purchasing an other asset $Y$ which would be comonotonic with it. In other words: Two comonotonic acts with value in $\mathbb{R}$ cannot be used for hedging purposes (hedging).

Let us illustrate this notion with the help of the following example. Consider the five mappings defined on the space $S=\left\{s_{1}, s_{2}, s_{3}\right\}$ by:

\footnotetext{
${ }^{1}$ Note that the terminology of comonotony comes from common monotony
} 


$\begin{array}{cccc} & s_{1} & s_{2} & s_{3} \\ & & & \\ X_{1} & 1 & 2 & 3 \\ X_{2} & -5 & 0 & 9 \\ X_{3} & 10 & 5 & 0 \\ X_{4} & 7 & 3 & 5 \\ X_{5} & 2 & 2 & 2\end{array}$

Every mapping being comonotonic with a constant mapping, $X_{5}$ is comonotonic with all other decisions. The decisions $X_{1}$ and $X_{2}$ are comonotonic. $X_{1}$ and $-X_{3}$ are comonotonic (one also says that $X_{1}$ and $X_{3}$ are anti-comonotonic). $X_{4}$ presents no comonotonic relation with $X_{1}, X_{2}$ or $X_{3}$. Note that comononotonicity is not transitive: $X_{4}$ is comonotonic with $X_{5}, X_{5}$ is comonotonic with $X_{1}$ and nevertheless $X_{4}$ and $X_{1}$ are not comonotonic (for more information on comonotony, see Denneberg (1994), Chateauneuf, Cohen and Kast (1997)).

\subsubsection{The Choquet integral}

In order to understand the models of decision under uncertainty that we will develop in the following section, let us give the definition of the Choquet integral (Choquet (1953) by first giving the definition of a capacity:

Definition $3 A$ (normalized) capacity $v$ on $(S, \mathcal{E})$ is a set-function from $\mathcal{E}$ to $[0,1]$ satisfying $v(\phi)=0, v(S)=1$ and monotone i.e. :

$\forall A, B \in \mathcal{E}, A \subset B \Rightarrow v(A) \leq v(B)$;

$A$ capacity is said to be convex if :

$\forall A, B \in \mathcal{E}, v(A \cup B)+v(A \cap B) \geq v(A)+v(B)$.

Another usual denomination is supermodular or monotone of order $2 \mathrm{ca}$ pacity.

When $S$ is finite, one considers $\mathcal{E}=2^{S}$.

Definition 4 For any measurable mapping $X$ from $(S, \mathcal{E})$ to $\mathbb{R}$, the Choquet integral $\int_{C h} X d v$ is defined by:

$$
\int_{C h} X d v=\int_{-\infty}^{0}[v(X>t)-1] d t+\int_{0}^{\infty} v(X>t) d t
$$

- First note that Choquet formula remains unchanged if strict inequalities are replaced by weak ones.

- Note also that if $v$ is a probability measure $P, \int_{C h} X d v$ reduces to $\int X d P=E_{P}(X)$, mathematical expectation of $X$ with respect to $P$. 
- When $X$ takes only a finite number of values, one can write :

$X=\left(x_{1}, A_{1} ; \ldots ; x_{i}, A_{i} ; \ldots ; x_{n}, A_{n}\right)$ where $x_{i} \in \mathbb{R}, x_{1} \leq \ldots \leq x_{i} \leq \ldots \leq$ $x_{n}$, and where $A_{i} \in \mathcal{E},\left(A_{i}\right)$ is a partition of $S$, the Choquet integral of $X$ is then given by:

$\int_{C h} X d v=x_{1}+\left(x_{2}-x_{1}\right) v\left[X \geq x_{2}\right]+\ldots+\left(x_{n}-x_{n-1}\right) v\left[X \geq x_{n}\right]$.

- Let $\mathcal{C}$ be a set of consequences, $f$ be a mapping from $\mathrm{S}$ in $\mathcal{C}, u$ a nondecreasing mapping from $\mathcal{C}$ in $\mathbb{R}$, by setting $X=u \circ f, X$ is a mapping from $S$ to $\mathbb{R}$, therefore one can define for given $u$ and $v$ :

$$
\int_{C h} u(f) d v=\int_{-\infty}^{0}[v(u(f)>t)-1] d t+\int_{0}^{\infty} v(u(f)>t) d t
$$

that we will call the Choquet expected utility -CEU- of the act $f$, $C E U(f)$ and that we will find again in the following sections.

\subsection{Characterization of the Choquet integral}

Schmeidler (1986) gave a characterization of functionals which are Choquet integrals and this characterization will be a crucial tool for the decision model initiated by Schmeidler and more generally for all Choquet expected utility models (CEU).

Characterization of the Choquet integral, 1986 :

Theorem 1 Let us consider the functional $I: \mathbb{X} \rightarrow \mathbb{R}$ satisfying $I\left(1_{S}\right)=1$ and the two following conditions:

(i) Comonotonic additivity : $X$ and $Y$ of $X$ comonotonic imply $I(X+Y)=$ $I(X)+I(Y)$ (hence $I(0)=0)$;

(ii) Monotonicity $: X \geq Y$ on $S$ implies $I(X) \geq I(Y)$;

Then by setting $v(A)=I\left(1_{A}\right)$ on $\mathcal{E}$, one gets for all $X$ in $\mathbb{X}$ :

$I(X)=\int_{C h} X d v$, or equally, $I(X)=\int_{-\infty}^{0}[v(X>t)-1] d t+\int_{0}^{\infty}[v(X>t)] d t$.

Conversely, any Choquet integral $I: x \in \mathbb{X} \rightarrow I(X)=\int_{C h} X d v \in \mathbb{R}$ satisfies $I\left(1_{S}\right)=1$ and conditions (i) et (ii).

This theorem is at the root of decision models under uncertainty based on Choquet integral.

\section{Decision under uncertainty}

Let us recall that by decision under uncertainty, we mean, in contrast with decision under risk, situations when there does not exist a given objective probability distribution on the set $S$ of states of the world, available to the decision maker. We saw in chapter 2 of this volume that the classical SEU 
model of decision under uncertainty imposes the use of a probabilistic representation, imposing that any situation of uncertainty should become a situation of subjective risk. This model contradicts the observed behaviors as shown by Ellsberg's Paradox.

\subsection{Ellsberg's paradox}

According to the importance of Ellsberg's Paradox in order to construct extensions of the classical models, we just recall it here, although it has already been made explicit in chapter 2 of this volume. Ellsberg proposes to subjects the following situation: an urn contains 90 balls, whose 30 are red $(R)$ and whose 60 are blue (B) or yellow (Y), in unknown proportion. So the number of blue balls may be from 0 to 60 and the complement consists of yellow balls. One will draw (at random) one ball from the urn and one asks the subjects to choose between the two following decisions: bet on $(R)$ (decision $X_{1}$ ) or bet on $(B)$ (decision $X_{2}$ ), then, independently, to choose between the following decisions: bet on $(R \cup Y)$ (decision $\left.X_{3}\right)$ or bet on $(B \cup Y)$ (decision $X_{4}$ ).

\subsubsection{Interpretation of Ellsberg's paradox in the framework of Sav- age}

Table 3.1 gives, for each decision the corresponding values of consequences (expressed in euros) of each decision according to the occurred event.

\begin{tabular}{|c|c|c|c|}
\hline & $30 / 90$ & & /90 \\
\hline & $\overbrace{R e d}$ & $\overparen{\text { Blue }}$ & Yellow \\
\hline & $R$ & $B$ & $J$ \\
\hline$X_{1}$ & 100 & 0 & 0 \\
\hline$X_{2}$ & 0 & 100 & 0 \\
\hline$X_{3}$ & 100 & 0 & 100 \\
\hline$X_{4}$ & 0 & 100 & 100 \\
\hline
\end{tabular}

Typically a majority of subjects make the following choices : $X_{1} \succ X_{2}$ and $X_{4} \succ X_{3}$, consequently, as it has been proved in the previous chapter, such a behavior is incompatible with the Savage Sure-Thing Principle, one of the major axiom of the theory.

Moreover, as noticed by Machina and Schmeidler (1992), such subjects are not even probabilistically sophisticated : this means that they do not ascribe subjective probabilities $p_{R}, p_{B}, p_{Y}$ to states of nature (i.e. elementary events $R, B, Y)$ and then use first order stochastic dominance axiom ${ }^{2}$ - a widely

\footnotetext{
${ }^{2}$ Let us recall that, if $X$ and $Y$ are real random variables, the first order stochastic dominance rule stipulates that if $\forall t \in \mathbb{R}, P\{X \geq t\} \geq P\{Y \geq t\}$, then $X$ should be weakly preferred to $Y$, the preference becoming strict if $P\{X \geq t\}>P\{Y \geq t\}$, for some $t_{0} \in \mathbb{R}$.
} 
accepted rule for partially ordered random variables. Otherwise, $g_{1} \succ g_{2}$ would imply $p_{R}>p_{B}$ and $g_{4} \succ g_{3}$ would imply $p_{B}+p_{Y}>p_{R}+p_{Y}:$ a contradiction.

\subsubsection{Interpretation of Ellsberg's paradox in Anscomb and Au- mann framework}

In the previous presentation of Ellsberg paradox, uncertainty is modelled through the set of the states of the world $S^{\prime}=\{R, B, J\}$ and bets are interpreted as mappings $X: S^{\prime} \rightarrow \mathbb{R}$.

We have seen, in chapter 2 of this volume, that Ellsberg's paradox is robust when, as Schmeidler (1989), one considers Ellsberg's experiment in the context of Anscombe $\& 3$ Aumann (1963) : actually it turns out that the independence axiom AA3 is violated.

Uncertainty now concerns the composition of the urn: the set $S$ of states of nature is composed of 61 states : $S=\left\{s_{0}, s_{1}, \ldots, s_{k}, \ldots, s_{60}\right\}$, where a state $s_{k}$ stands for a given composition of the urn : "30 red balls, $k$ blue balls and $60-k$ yellow balls ".

Let us call $\mathcal{Y}$ the set of all lotteries on $\mathcal{C}=\{0,100\}$, or equally of all probability distributions on $\mathcal{C}$ with finite support. The uncertain prospect described by the act $X_{i}$ in the Savage framework is now characterized in the framework of Anscombe and Aumann, by the mapping $g_{i}$ from $S$ to $\mathcal{Y}, g_{i}$ : $S \rightarrow \mathcal{Y}$ in the following way:

To each state of nature $s_{k}$ of $S$, the consequence $g_{i}\left(s_{k}\right)$ is the lottery : $\left(X_{i}(R), 30 / 90 ; X_{i}(B), k / 90 ; X_{i}(J),(60-k) / 90\right)$, or equally the lottery offering $X_{i}(R)$ with probability $\frac{30}{90}, X_{i}(B)$ with probability $\frac{k}{90}$, and $X_{i}(J)$ with probability $(60-k) / 90$ (see table 2.2 linked with the various acts in chapter 2 of this volume, paragraph 2.4.4).

If one assumes, as implicitly done by Schmeidler and Anscombe-Aumann that, under risk, the decison maker maximizes an expected utility (see Chapter 1 of this volume) with a von Neumann utility function $u$ (which can be assumed without loss of generality such that $u(0)=0, u(100)=1$ ), one can also establish through a direct computation that, in the Anscombe-Aumann framework, the expected utility model under uncertainty cannot explain preferences described above : actually imagine that the decision-maker ascribes probabilities to the events $s_{k}$, and that he behaves in accordance with the Anscombe-Aumann expected utility model, i.e. prefers $h$ to $g$ if and only if $\sum p_{k} u\left(h\left(s_{k}\right)\right) \geq \sum p_{k} u\left(g\left(s_{k}\right)\right)$. Then, a simple computation shows that $g_{1} \succ g_{2}$ would give $30>\sum k p_{k}$ while $g_{4} \succ g_{3}$ would give $30<\sum k p_{k}$, a contradiction.

\subsection{Schmeidler's model in Anscombe-Aumann's framework}

In order to explain such "paradoxes" and to separate perception of uncertainty from valuation of outcomes, Schmeidler (1989) has proposed a model 
which relaxes the usual independence condition while offering a flexible but simple formula. As was previously pointed out, Schmeidler $(1982,1989)$ has developed his model in the Anscombe-Aumann's framework. Hence, in this section, the set $S$ of sets of nature is finite and the events are the elements of $\mathcal{E}=2^{S}$. The set of consequences $Y$ is the set of lotteries on a given set of outcomes $\mathcal{C}$ (i.e. $\mathcal{Y}$ is the set of probability distributions on $\mathcal{C}$ with finite support). The set of acts is the set of mappings $\mathbb{F}_{0}$ from $S$ to $\mathcal{Y}$ also called "horse lotteries". Let $\succsim$ be the preference relation of the decision-maker on the set $\mathbb{F}_{0}$.

\subsubsection{Comonotonic independence}

Let us recall that, in Anscombe-Aumann framework, the subjective expected utility model, SEU (see Chapter 2 of this volume), obtains mainly through the following axiom:

Axiom 1 Independence axiom (Anscombe and Aumann)

For all $f, g, h$ in $\mathbb{F}_{0}$, and for all $\alpha$ in $] 0,1[: f \succ g$ implies $\alpha f+(1-\alpha) h \succ$ $\alpha g+(1-\alpha) h$.

We have seen that a great majority of behaviors contradict such an axiom (Interpretation of Ellsberg's paradox in Anscomb and Aumann). In order to weaken this axiom, Schmeidler introduced the definition of comonotonic acts and then the following weakened axiom:

Axiom 2 Axiom of comonotonic independence (Schmeidler)

For all acts $f, g$ and $h$ in $\mathbb{F}_{0}$, pairwise comonotonic and for all $\alpha$ in ]0,1[: $f \succ g$ implies $\alpha f+(1-\alpha) h \succ \alpha g+(1-\alpha) h$.

Roughly speaking, comonotonic independence requires the direction of preferences to be retained, provided hedging effects are not involved. This intuition which is crucial in Schmeidler's model, will appear more transparent in Schmeidler's representation theorem (1989).

By adding to this key axiom, some usual axioms as weak order and continuity, Schmeidler (1989) derives the characterization of his model where typical preferences observed in paragraph 3.2.1 become admissible.

\subsubsection{Representation of preferences by a Choquet integral in Anscombe- Aumann's framework}

Schmeidler shows that the preference relation $\succsim$ on $\mathbb{F}_{0}$ (the acts in AnscombeAumann's framework) satisfying the axioms previously described is represented by a Choquet integral with respect to a unique capacity $v$. More precisely,

For all $f$ and $g$ in $\mathbb{F}_{0}: f \succsim g$ if and only if $\int_{C h} u(f()) d. v \geq \int_{C h} u(g()) d$. $\mathcal{C}$

where $u$ is the von Neumann utility function on the set $\mathcal{Y}$ of lotteries on 
Notice that capacity $v$ is substituted to probability $P$ in Anscombe-Aumann's theorem.

The strategy of Schmeidler's proof consists in first noting that axiom 2 entails axiom 1 on the set of constant acts $\mathbb{F}_{0}^{c}$, hence the existence of a vNM utility function $u$ on the set $\mathcal{Y}$ of lotteries, and therefore the ability of linking in a natural way any act $f=\left(y_{1}, A_{1}, \ldots y_{n}, A_{n}\right)=\sum_{i=1}^{i=n} y_{i} 1_{A_{i}}$, where $y_{i} \in \mathcal{Y}$, with the real random variable $u(f)=\sum_{i=1}^{i=n} x_{i} 1_{A_{i}}$, where $x_{i}=u\left(y_{i}\right), i=1, . . n$.

Then denoting by $\mathfrak{X}_{0}$ the set of such variables, Schmeidler shows in a second step that the preorder induced on $\mathfrak{X}_{0}$, denoted $\succsim_{0}$ is representable by a Choquet integral, equally, that there exists a capacity v on $\mathrm{S}$ such that:

$$
\forall(X, Y) \in \mathfrak{X}_{0}^{2}, X \succsim_{0} Y \Longleftrightarrow \int_{C h} X d v \geq \int_{C h} Y d v
$$

\subsection{Choquet expected utility (CEU) models in Savage's frame- work}

By Choquet expected utility (CEU) models, we mean those non-additive models directly connected with the Choquet integral which, following pioneer's work of Schmeidler $(1982,1989)$ in the Anscombe-Aumann framework, have been derived in Savage framework as for example by Gilboa (1987) or Wak$\operatorname{ker}(1990)$.

Savage framework seems more natural than Anscombe-Aumann one's where consequences are lotteries but there the axiomatization becomes more sophisticated.

Although Savage framework allows for more general consequence sets $\mathcal{C}$, we will confine in this paragraph 2.3. to $\mathcal{C}=\mathbb{R}$, which permits a simple exposure of the main properties of CEU models.

So we consider a decision-maker making his choices inside the set $\mathbb{X}$ of acts consisting of all functions $X:(S, \mathcal{E}) \rightarrow \mathbb{R}, \mathcal{E}$-measurable and bounded where $S$ is a set of states of nature and $\mathcal{E}$ a $\sigma$-algebra of subsets of $S$. This decision-maker is in a situation of uncertainty, and $\succsim$ is a preference relation on $\mathbb{X}$.

\subsubsection{Simplified version of Schmeidler's model in Savage's frame- work}

In this framework, referred to as Savage's framework, and which fits the first and simple presentation of Ellsberg's paradox, a simplified translation of the comonotonic independence axiom of Schmeidler is as follows :

Axiom 3 Axiom of comonotonic independence (Chateauneuf, (1994)):

$$
\begin{aligned}
\text { Let } X, Y,, Z & \in \mathbb{V}, X \text { et } Z \text { comonotonic, } Y \text { and } Z \text { comonotonic, } \\
\text { then } X & \sim Y \Rightarrow X+Z \sim Y+Z
\end{aligned}
$$


where for $\mathcal{C}=\mathbb{R}$, the definition of comonotonic acts is the one of definition 2 .

Axiom 3.3?? of comonotonic independence requires to maintain the direction of preferences when adding the same act, as soon as no asymmetric reduction of uncertainty is involved through hedging effects. On the contrary, in case of asymmetric reduction of uncertainty (through hedging effects), axiom 3.3?? allows for modifying the direction of preferences.

Example 3.1 below shows how such a behavior under uncertainty can be taken into account in a case where the acts give results depending of the realization of the event $A$ or of the complementary event $\bar{A}$.

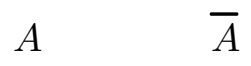

$\begin{array}{llll}\text { Example 1 } & X & 25000 & 15000 \\ & Y & 12000 & 30000 \\ & Z & 15000 & 25000 \\ & X+Z & 40000 & 40000 \\ & Y+Z & 27000 & 55000\end{array}$

Assume at the beginning indifference between $X$ and $Y(X \sim Y) . Z$ is comonotonic with $Y$ but not with $X, Z$ may be used as a hedge against $X$ but not against $Y$, and consequently an uncertain averse decision-maker may express after addition of the variable $Z$, the following strict preference : $X+$ $Z \succ Y+Z$.

Under the key comonotonic independence axiom and other classical axioms as weak order and continuity, it is then possible to deduce from these axioms a simplified version of Schmeidler's model where preferences can be represented by a Choquet integral with respect to a capacity $v$, i.e.:

For all $X, Y \in X, X \succsim Y$ if and only if $\int_{C h} X d v \geq \int_{C h} Y d v$ (see Chateauneuf(1994)).

Note that this model is simplified in the sense that utility of outcomes is linear, a consequence of the independence axiom of Chateauneuf (1994).

Such a result is deduced from the fundamental theorem of Schmeidler (1986), which characterizes the Choquet integral (1953)), and appears as a crucial tool for Schmeidler's model and more generally for Choquet expected utility (CEU) models .

\subsubsection{Choquet expected utility model in Savage's framework}

When utility of results is no longer necessarily linear, one gets the following classical definition of the Choquet expected utility model:

Definition 5 A decision-maker satisfies the Choquet expected utility (CEU) model if the decision-maker's preferences on the set of acts $\mathbb{V}$ can be represented 
with the help of a utility function under certainty $u: \mathbb{R} \rightarrow \mathbb{R}$, non-decreasing and defined up to an increasing affine transformation and with the help of a personal evaluation of the likelihood of events through a capacity $v$. Preferences representation is given by $I(u(X))=\int_{C h} u(X) d v$, the Choquet integral of $u(X)$ with respect to capacity $v$, defined for $X \in \mathbb{X}$ by

$$
\int_{C h} u(X) d v=\int_{-\infty}^{0}[v(u(X)>t)-1] d t+\int_{0}^{\infty}[v(u(X)>t)] d t
$$

Note that this CEU model generalizes equation (1) with a function $u$ which is not necessarily linear.

For a simple step act $X=\left(x_{1}, A_{1} ; \ldots ; x_{n}, A_{n}\right)=\sum_{i=1}^{i=n} x_{i} 1_{A_{i}}$ where $x_{i} \in \mathbb{R}$, $A_{i} \in \mathcal{E},\left(A_{i}\right)$ partition of $S$, one obtains :

$$
\begin{gathered}
\int_{C h} u(X) d v= \\
u\left(x_{1}\right)+\left(u\left(x_{2}\right)-u\left(x_{1}\right)\right) v\left[X \geq x_{2}\right]+\ldots+\left(u\left(x_{n}\right)-u\left(x_{n-1}\right)\right) v\left[X \geq x_{n}\right] .
\end{gathered}
$$

Remark 1 One can interpret the behavior of a decision-maker using this model $\int_{C h} u(X) d v$ as follows: The decision-maker values $X$ by first evaluating the utility of the minimum result $x_{1}$ he gets with certainty, and then adding the additional increases of utility $u\left(x_{i+1}\right)-u\left(x_{i}\right), 1 \leq i \leq n-1$, weighted by his personal belief $v\left[X \geq x_{i+1}\right]$ of their occurence.

Example of computation of such a Choquet integral Let $S=\left\{s_{1}, s_{2}, s_{3}\right\}$.

Let $v$ be a capacity on $S$ as below and $X$ an act such that the values of $u(X)$ are given in the following table:

$$
\begin{array}{ccccccccc} 
& \phi & s_{1} & s_{2} & s_{3} & s_{1} \cup s_{2} & s_{1} \cup s_{3} & s_{2} \cup s_{3} & S \\
v & 0 & 1 / 3 & 0 & 0 & 1 / 3 & 1 / 3 & 2 / 3 & 1 \\
u(X) & & a & b & c & & & &
\end{array}
$$

The evaluation of the Choquet integral $I=\int_{C h} u(X) d v$ depends of the ranking of $a, b, c$ :

- $\quad$ if $a<b<c, \int_{C h} u(X) d v=a+(b-a) v\left(\left\{s_{2}, s_{3}\right\}\right)+(c-b) v\left(\left\{s_{3}\right\}\right)$

$$
=1 / 3 a+2 / 3 b
$$

- $\quad$ if $c<a<b, \int_{C h} u(X) d v=2 / 3 c+1 / 3 a$

- $\quad$ if $b<c<a, \int_{C h} u(X) d v=2 / 3 b+1 / 3 a$

A classical integral, with an additive measure, would naturally take the same value whatever be the ranking of consequences.

We will now give the key axiom of CEU theory. 


\subsubsection{The comonotonic sure thing principle}

The main feature of the CEU model is to allow taking into account possible hedging effects. For this purpose, the crucial axiom in the axiomatization of CEU, is the comonotonic sure thing principle (see for instance, Gilboa (1987), Chew and Wakker (1996)), a weakening of Savage's sure thing principle, which can be stated in the following way :

Axiom 4 The comonotonic sure thing principle

Let $X=\sum_{i=1}^{n} x_{i} 1_{A_{i}}$ et $Y=\sum_{i=1}^{n} y_{i} 1_{A_{i}}$, where $\left\{A_{i}\right\}$ is a partition of $S$ and $x_{1} \leq \ldots \leq x_{i} \leq \ldots \leq x_{n} ; y_{1} \leq \ldots \leq y_{i} \leq \ldots \leq y_{n}$ are such that $x_{i_{0}}=y_{i_{0}}$ pour some $1 \leq i_{0} \leq n$. Then $X \succsim Y$ implies $X^{\prime} \succsim Y^{\prime}$ for the acts $X^{\prime}$ et $Y^{\prime}$ obtained

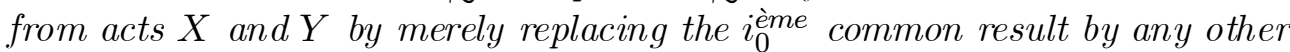
common result which preserves the ranking $i_{0}$ for both acts $X$ and $Y$.

This axiom expresses that, as long as acts remain comonotonic (i.e., no hedging effect happens), there is no reason to change the direction of preferences when a common outcome is modified.

Note, however, that even jointly with standard axioms of weak order, continuity and monotonicity, the comonotonic sure-thing principle fails to fully characterize CEU.

For instance, Wakker (1989) completes the axiomatization of the CEU model by strengthening axiom 3.4 to a "comonotonic trade-off consistency" one.

We now turn to the ability of Schmeidler's model to handle uncertainty aversion (and symmetrically uncertainty appeal).

\subsection{Uncertainty aversion}

In his seminal papers, Schmeidler $(1982,1989)$ has shown the great ability of his model to capture the concept of uncertainty aversion. He defined uncertainty aversion through convexity of preferences i.e. : $\forall f, g \in \mathbb{F}_{0}, \forall \alpha \in$ $[0,1], f \sim g \Rightarrow \alpha f+(1-\alpha) g \succsim f$, interpreting this axiom as "smoothing" or averaging potential outcomes makes the DM better off. This definition revealed as particularly meaningful since as proved by Schmeidler $(1986,1989)$, uncertainty aversion is equivalent to the capacity $v$ being convex and since furthermore one has :

Proposition 2 Schmeidler(1986)

Let $I: \mathbb{X} \rightarrow \mathbb{R}$ be a Choquet integral with respect to a capacity $v$, i.e. $\forall X \in$ $\mathbb{X}, I(X)=\int_{C h} X d v$, then the two following conditions are equivalent:

(i) $v$ is convex;

(ii) Core $(v) \neq \phi$ where core $(v)=\left\{\begin{array}{c}\text { simply additive probabilitiesPonE } \\ \text { t.q. } P(A) \geq v(A), \forall A \in \mathcal{E}\end{array}\right\}$ and for all $X$ in $\mathbb{X}: \int_{C h} X d v=\operatorname{Min}\left\{\int X d P, P \in \operatorname{core}(v)\right\}$. 
This proposition offers an attractive interpretation of uncertainty aversion in terms of pessimism : in Schmeidler's model, an uncertainty averse decisionmaker, behaves in the following way: he considers for every act, among all probability distributions $P$ in core of $v$, the one giving the minimum expected utility $E_{P} u(f)$ of this act, and then chooses the act which maximizes this minimum :

$$
\text { i.e. } \forall f, g \in \mathbb{F}_{0}, f \succsim g \text { iff } \underset{P \in \operatorname{core}(v)}{\operatorname{Min}} \int_{S} u(f) d P \geq \underset{P \in \operatorname{Min}(v)}{\operatorname{Min}} \int_{S} u(g) d P \text {. }
$$

Such an interpretation would remain true for the CEU model (i.e. in Savage's framework) since for such a model, convexity of preferences is equivalent to $v$ convex and $u$ concave (see Chateauneuf and Tallon (2002)).

Moreover, in the simple case of the CEU model with constant marginal utility $(u(x)=x, \forall x \in \mathbb{R})$, one can give directly an interpretation in terms of hedging effects, since there, convexity of preferences is equivalent to the following uncertainty aversion axiom (Chateauneuf (1994)):

\section{Axiom 5 Uncertainty aversion ${ }^{3}$}

For $X, Y, Z \in \mathbb{X}, Y$ et $Z$ comonotonic, then $X \sim Y \Rightarrow X+Z \succsim Y+Z$

Notice that this uncertainty aversion axiom implies the comonotonic independence axiom and therefore characterizes the simplified Schmeidler's model, where, moreover, $v$ is convex.

This axiom allows for taking into account hedging effects : since $Z$ is not a hedge against $Y$ but may be a hedge against $X$, hence $X+Z$ may display a reduction of uncertainty with respect to $Y+Z$, and therefore $X+Z$ may be preferred to $Y+Z$ by an uncertainty averse DM.

Such an interpretation fits particularly well for interpreting behaviors in Ellsberg's example : let us describe uncertainty in Ellsberg's example -in accordance with paragraph 3.2.1- by : $S=\{R, B, Y\}, \mathcal{E}=2^{S}$. Let $\mathcal{P}$ be the set of all probability distributions on $\left(S, 2^{S}\right)$ compatible with the information (i.e. $\mathcal{P}=\left\{\right.$ probability distributions on $\left(S, 2^{S}\right)$ such that $\left.P(R)=1 / 3\right\}$ and let $v$ defined by $v(A)=\operatorname{Inf}_{\mathcal{P}} P(A), \forall A \in \mathcal{E} ;$ one obtains table 3.3.??

$$
\begin{array}{rrrrrrrrr} 
& \phi & R & B & Y & R \cup B & R \cup Y & B \cup Y & S \\
v & 0 & 1 / 3 & 0 & 0 & 1 / 3 & 1 / 3 & 2 / 3 & 1
\end{array}
$$

It is straightforward to show that $v$ is a convex capacity ${ }^{4}$ and that $\mathcal{P}=$ $\operatorname{core}(v)$.

\footnotetext{
3 a completer

${ }^{4} v$ is actually an infinite monotone capacity or else a belief function
} 
Let us compute $I(X)=\int_{C h} X d v$ for all considered acts :

$I\left(X_{1}\right)=1 / 3 \times 100>I\left(X_{2}\right)=0 \times 100$, thus $X_{1} \succ X_{2}$

$I\left(X_{4}\right)=2 / 3 \times 100>I\left(X_{3}\right)=1 / 3 \times 100$, thus $X_{4} \succ X_{3}$

Consequently one can explain the behaviors in Ellsberg's paradox by uncertainty aversion.

\subsection{The multi-prior model}

We now consider the model "max-min" of Gilboa and Schmeidler (1989). In this model, the agents have a set of a priori probability laws (and not a single one as in the Bayesian paradigm ) and use the maximin criterion for evaluating decisions through this set of initial beliefs (multiple prior).

\subsubsection{The axiomatic of the model}

Gilboa and Schmeidler (1989) consider an Anscombe-Aumann framework (1963), where the set of consequences is a set $\mathcal{Y}$ of laws with finite support over a set $\mathcal{C}$. This axiomatic is very simple and leans mainly on the two following axioms. The first one is axiom 3.6?? of certainty independence:

Axiom 6 For all $f, g$ of $\mathbb{F}_{0}$ and $h$ constant decision of $\mathbb{F}_{0}$, for all $\left.\alpha \in\right] 0,1[$

$$
f \succ g \Longrightarrow \alpha f+(1-\alpha) h \succ \alpha g+(1-\alpha) h
$$

This axiom is weaker than the usual independence axiom, since it applies only when adding a "common consequence" which is constant. This axiom is implied by the comonotonic independence axiom (axiom 3). The second axiom is the one of uncertainty aversion previously defined in Schmeidler's model(1989) :

Axiom 7 For all $f, g$ in $\mathbb{F}_{0}$ and $\left.\alpha \in\right] 0,1[$

$$
f \sim g \Longrightarrow \alpha f+(1-\alpha) g \succsim f
$$

Proposition 3 (Gilboa and Schmeidler (1989)) Under the axiom of weak order, an axiom of monotony, an axiom of continuity and the axioms 6 and 7 , there exists a set of probability measures $\mathcal{P}$, closed and convex, and a utility function u of von Neumann: $\mathcal{Y} \rightarrow \mathbb{R}$ such that:

$$
f \succsim g \Longleftrightarrow \min _{P \in \mathcal{P}} \int u(f) d P \geq \min _{P \in \mathcal{P}} \int u(g) d P
$$

THe function $u$ is unique up to a positive affine transformation, while the set $\mathcal{P}$ is unique if closed in the weak-star topology. 
The interpretation of this representation is fairly simple. The decisionmaker behaves as if he had a set of a prior beliefs (instead of a unique one as in the expected utility model). In order to evaluate an act, he computes the expected utility of this act with respect to all probability distributions that he considers in $\mathcal{P}$, and then takes the minimum. This last operation fits to a pessimism attitude or uncertainty aversion. Note that by construction, this model can only take into account pessimistic behaviors and not "optimistic" behaviors or mixed behaviors.

\subsubsection{Comparing multi-prior model with Choquet utility model}

The multi-prior model is closely linked with the Choquet utility model. With this model, it is possible to interpret the Choquet capacity in terms of beliefs. Actually, from proposition 2 :

$$
v \text { is convex } \Longleftrightarrow\left\{\begin{array}{l}
\operatorname{core}(v) \neq \emptyset \text { and } \\
\int_{C h} u(X) d v=\min _{P \in \operatorname{core}(v)} \int u(X) d P \text { for all } X \in \mathbb{X}
\end{array}\right.
$$

When the decision-maker's capacity is convex, this decision maker behaves as in a multi-prior model whose set of probability measures is given by the core of the capacity. The multi-prior model allows to give an "objective" foundation to the (subjective) capacity of the Choquet utility model; capacity which then represents the lower envelope of the family of probability measures of the capacity of the multi-prior model. Nevertheless, one should notice that every closed and convex family of probability measures is not necessarily the core of a convex capacity and therefore that the multi-prior model is not a particular case of the Choquet utility case with a convex capacity. Moreover the behaviors described by a Choquet integral with respect to a non-convex capacity cannot be described by the multi-prior model.

Remark 2 The behavior of a decision-maker of the multi-prior type may be considered as excessively pessimistic. In fact, in the next section we present the models of Jaffray (1989a, 1989b) and Jaffray-Philippe (1997) which model less extreme behaviors.

\subsubsection{CEU model and lower and upper envelopes of a family of probability distributions}

Jaffray (1989a, 1989b) and Jaffray and Philippe (1997) have proven that, under some conditions, it was possible to write a Choquet integral with respect to any capacity $\mathrm{v}$ as a linear combination ?? of two terms :respectively the minimum and the maximum of expected utilities with respect to a family of probability distributions, the weight between the two representing an index of pessimism. 
As we showed previously, in the Ellsberg experiment, the uncertainty can be summarized by the lower envelope $v()=.\operatorname{Inf}_{P \in \mathcal{P}} P($.$) , and in this case, this$ capacity $v$ is convex, allowing thus simpler formula, as we showed in proposition 2. ; indeed, if $v$ is a convex capacity on $(S, \mathcal{E})$, then $\forall X \in \mathbb{X}, \min$ $\left\{\int X d P, P \in \operatorname{core}(v)\right\}=\int_{C h} X d v$.

Such situations of uncertainty, summarized by a lower envelope (i.e. a convex capacity) have been defined by Jaffray (1989a, 1989b) as being "regular uncertainty"

Definition 6 We are in a situation of regular uncertainty when the situation of uncertainty, defined by a family of probability distributions $\mathcal{P}$ on $(S, \mathcal{E})$ is completely characterized by its lower envelope $c$ where $c(A)=\operatorname{Inf}_{P \in \mathcal{P}} P(A)$ and $c$ is convex, meaning that $\mathcal{P}=\left\{P\right.$ on $\left.\left(S, 2^{S}\right), P \geq c\right\}$. We will denote by $C$ the upper envelope of $\mathcal{P}: C=\underset{P \in \mathcal{P}}{\operatorname{Sup}} P($.$) and C(A)=1-c(\bar{A}), \forall A \in \mathcal{E})$.

This "regular uncertainty" can be encountered in natural situations as shown by Dempster (1967). Let us assume (as in Dempster) that $(, 2, \pi)$ is a finite probability space and that $\Gamma$ is a correspondence from to $\mathcal{E}^{*}=\mathcal{E}-\{\phi\}$, where $\mathcal{E}=2^{S}$ and $S$ is a finite state space. Let us interpret $\Gamma$ as informing us that if $\varpi \in$ occurs, then the true state $s$ belongs to $\Gamma(\varpi)$ (such a state space $(, 2, \pi)$ is called a message space) ; one can then states that each event $A \in \mathcal{E}$ occurs with a probability at least equal to $v(A)$ where $v(A)=\sum_{B \subset A} m(B)$, and $m(B)=\sum_{\{\varpi \in, \Gamma(\varpi)=B\}} \Gamma(\{\varpi\})$; it can be shown that $v$ is a belief function (i.e. a particular case of a convex capacity (see for instance Shafer (1976) and chapter 3 in first volume??).

Now, in such situations of regular uncertainty, it can be the case that a CEU decision maker don't have necessarily uncertainty aversion (i.e. does not have necessarily a subjective assessment of events represented by a capacity $v=c$, but by a subjective assessment of events represented by a capacity $v=$ $\alpha c+(1-\alpha) C$ with $\alpha \in[0,1], c$ being convex.

Such a behavior where the value of $\alpha$ can be interpreted as the pessimism index due to Hurwicz, has been studied and axiomatized by Jaffray and Philippe (1997) who have shown that this behavior was compatible both with the CEU model and with the Jaffray (1989a,1989b).

\section{Decision under risk}

From now, we assume that there is an "objective" probability distribution $P$ on $(S, \mathcal{E})$ and that the decision maker knows it; we say then that the decision maker is facing a problem of decision under risk. 
Moreover, to make the exposition simpler, we suppose that the probability distribution $P$ is $\sigma$-additive and non-atomic (i.e. $\forall A \in \mathcal{E}$, such that $P(A)>0$, $\forall \alpha \in(0,1], \exists B \in \mathcal{E}, B \subset A$, such that $P(B)=\alpha P(A))$; thanks to these assumptions, the set $\mathbb{X}$ of acts generate any real bounded random variable.

Any element $X$ of $\mathbb{X}$ is then a random variable whose probability distribution is $P_{X}$. Let us denote $F_{X}$ the cumulative distribution function of $X$ $\left(\forall x \in \mathbb{R}, F_{X}(x)=P\{s \in S / X(s) \leq x\}=P_{S}\{X \leq x\}\right), E(X)$ its expected value and $\mathcal{L}$ the set of all probability distributions of elements of $\mathbb{X}$.

Since every $X$ of $\mathbb{X}$ induces a probability distribution $L(X)$ on $\mathbb{R}$, the preference relation $\succsim$ on $\mathbb{X}$ also induces a preference relation on $\mathcal{L}$ that (by misuse of notation) we also denote by $\succsim$, under the following condition $H_{0}$ :

Condition $H_{0}$ (Neutrality): Two random variables with the same probability distribution are always indifferent.

Hence, under this condition, any axiomatization on $(\mathbb{X}, \succsim)$ can be replaced by an axiomatization on $(\mathcal{L}, \succsim)$.

Remark 3 Any discrete act $X$ of $\mathbb{X}$ can be written : $X=\left(x_{1}, A_{1} ; \ldots ; x_{k}, A_{k}\right.$; $\left.\ldots ; x_{n}, A_{n}\right)$, where $\left(A_{i}\right)(i=1, \ldots, n)$ is a partition of $S$ and $x_{i}$ the consequence of $X$ on each $A_{i}$. Under risk, the probability distribution of this random variable will be denoted : $L(X)=\left(x_{1}, p_{1} ; \ldots ; x_{k}, p_{k} ; \ldots ; x_{n}, p_{n}\right)$ with $x_{1} \leq x_{2} \leq$ $\cdots \leq x_{n}, p_{i}=P\left(A_{i}\right) \geq 0$, and $\sum p_{i}=1$.

In the following, it can be useful to use also the following notation :

$$
L(X)=\left(x_{1}, 1-q_{1} ; x_{2}, q_{1}-q_{2} ; \ldots ; x_{n-1}, q_{n-2}-q_{n-1} ; x_{n}, q_{n-1}\right)
$$

where, for $i=1, \ldots n-1, q_{i}=\sum_{j=i+1}^{j=n} p_{j}$.

In this section, we identify any consequence $c$ with its Dirac probability distribution $\delta_{\{c\}}$.

\subsection{EU model and Allais paradox}

We have study in detail, in chapter 1 of this volume, the classical model of decision under risk : the Expected Utility (EU) model. As early as 1953, Allais has built a couple of alternatives for which a majority of subjects, confronted with that choices, choose in contradiction with the independence axiom and thus in violation with the EU model (see chapter 1, section 1.4.1.).

Since this experiment, known as Allais "paradox", has been a cornerstone to question the EU model, let us first recall, in this chapter, the original Allais paradox (Allais (1953)).

Subjects were asked to choose between the following lotteries (say in thousand euros):

$L_{1}$ : win $1 M$ with certainty or $L_{2}$ : win $1 M$ with probability $0.89,5 M$ with probability 0.10 , and 0 with probability 0.01 
and then independently, to choose between the following lotteries:

$L_{1}^{\prime}$ : win $1 M$ with probability 0.11 and 0 with probability 0.89 or

$L_{2}^{\prime}$ : win $5 M$ with probability 0.10 and 0 with probability 0.90 .

Most subjects choose $L_{1}$ over $L_{2}$, and $L_{2}^{\prime}$ over $L_{1}^{\prime}$. These simultaneous choices violate the independence axiom. Indeed, defining $P$ as the lottery yielding $1 M$ with probability 1 and $Q$ as the lottery yielding 0 with probability $1 / 11$ and $5 M$ with probability $10 / 11$, one can check that:

$$
\begin{aligned}
& L_{1}=0,11 P+0,89 \delta_{1} \\
& L_{2}=0,11 Q+0,89 \delta_{1} \\
& L_{1}^{\prime}=0,11 P+0,89 \delta_{0} \\
& L_{2}^{\prime}=0,11 Q+0,89 \delta_{0}
\end{aligned}
$$

where $\delta_{0}$ is the lottery "win 0 with probability 1 " and $\delta_{1}$ is the lottery "win $1 M$ with probability 1 ". The observed choices are thus in contradiction with the independence axiom ${ }^{5}$.

This experiment has been ran many times, on various populations of subjects with similar results: about $66 \%$ of the choices are in contradiction with the independence axiom.

Not only observed behaviors are in contradiction with EU theory, but also the EU model raised a theoretical difficulty, namely, the interpretation of the function $u$ (called von Neumann's utility) characterizing the DM's behavior : as pointed out by Allais himself, the function $u$ has, in fact a double role of expressing the DM's attitude with respect to risk (concavity of $u$ implying risk aversion) and the DM's valuation of differences of preferences under certainty (concavity of $u$ implying then diminishing marginal utility of wealth). These evidences have led researchers to built more flexible models. The RDU model that we will present in the next section, not only will disentangle attitude towards risk and satisfaction of outcomes, but also will be compatible with observed behaviors in Allais experiment.

\subsection{The Rank Dependent Expected Utility model}

\subsubsection{Definition of the Rank Dependent Expected Utility model}

The Rank Dependent Expected Utility (RDU) model is due to Quiggin (1982) under the denomination of "Anticipated Utility". Variants of this model are due to Yaari (1987), Segal $(1987,1993)$ and Allais (1988). More general axiomatizations can be found in Wakker (1994), Chateauneuf (1999).

Definition $7 A D M$ behaves in accordance with the rank-dependent expected utility $(R D U)$ model if the DM's preferences on $(\mathcal{L}, \succeq)$ are characterized by two

\footnotetext{
${ }^{5}$ since under independence axiom:

$L_{1} \succeq L_{2} \Longrightarrow P \succeq Q \Longrightarrow L_{1}^{\prime} \succeq L_{2}^{\prime}$
} 
functions $u$ and $f:$ a continuous, increasing, cardinal $l^{6}$ utility function $u: \mathbb{R} \rightarrow$ $\mathbb{R}$ (that plays the role of utility on certainty) and an increasing probability -transformation function $f:[0,1] \rightarrow[0,1]$ that satisfies $f(0)=0, f(1)=1$. Such a DM prefers the random variable $X$ to the random variable $Y$ if and only if $V(X) \geq V(Y)$, where the functional $V$ is given by :

$$
V(Z)=V_{u, f}(Z)=\int_{-\infty}^{0}[f(P(u(Z)>t))-1] d t+\int_{0}^{\infty} f(P(u(Z)>t)) d t .
$$

- If the transformation function $f$ is the identity function $f(p) \equiv p$, then $V(Z)=V_{u, I}(Z)$ is the expected utility $E[u(Z)]$ of the random variable $Z$.

- If the utility $u$ is the identity function $u(x) \equiv x$, then $V(Z)=V_{I, f}(Z)$ is theYaari functional (see Yaari (1987)). In fact, Yaari axiomatized independently his model ${ }^{7}$.

- If both transformation and utility are identity functions, then $V(Z)=$ $V_{I, I}(Z)$ is simply the expected value $E[Z]$ of the random variable $Z$.

When $Z$ is discrete, $V(Z)$ can be written as

$V(Z)=u\left(x_{1}\right)+f\left(q_{1}\right)\left[u\left(x_{2}\right)-u\left(x_{1}\right)\right]+. .+f\left(q_{2}\right)\left[u\left(x_{3}\right)-u\left(x_{2}\right)\right]+\cdots+f\left(q_{n-1}\right)\left[u\left(x_{n}\right)-u\left(x_{n-1}\right]\right.$

We can then interpret the evaluation of a RDU decision maker: he evaluate first, for sure, the utility of the worst outcome $u\left(x_{1}\right)$ and then, weights the additional possible increases of utility $u\left(x_{i}\right)-u\left(x_{i-1}\right)$ by his personal transformation $f\left(q_{i}\right)$ of the probability $v_{i}$ of having at least $x_{i}$.

According to this interpretation, if the decision maker behaves in such a way that $f(p) \leq p$, it means that he underestimates all the additional utilities of gains. In this sense, we will call him pessimistic under risk. In the same way, $u$ reflecting now his satisfaction for wealth, concavity of $u$ reveals diminishing marginal utility.

Remark 4 Let us notice that various attempts to generalize EU model by a functional likewise:

$\left(x_{1}, p_{1} ; \ldots ; x_{k}, p_{k} ; \ldots ; x_{n}, p_{n}\right) \longmapsto \sum f\left(p_{i}\right) u\left(x_{i}\right)$ with $f:[0,1] \longrightarrow[0,1]$ and $f(0)=0, f(1)=1$, failed because the only functionals compatible with the first order stochastic dominance is obtained for $f(p)=p$, meaning that this functional reduce to EU.

\footnotetext{
${ }^{6} u$ is cardinal if it is defined up to an affine increasing transformation.

${ }^{7}$ Yaari model is called "Dual Theory". This model is as parcimonious as EU model since it used only one function $f$; however, this model allows to distinguish strong risk aversion from weak risk aversion, which is not possible in EU model.
} 
Allais "Paradox" is compatible with RDU model As an exercise, we can evaluate in a RDU model the different lotteries of Allais example. setting (w.l.o.g.) $u(0)=0$; we have

$V\left(L_{1}\right)=u(1) ; V\left(L_{2}\right)=u(1)(f(0.99)-f(.0 .10))+u(5) f(0.10) ;$

$V\left(L_{1}^{\prime}\right)=u(1) f(0.11)$ and $V\left(L_{2}^{\prime}\right)=u(5) f(0.10)$.

$L_{1} \succ L_{2}$ implies $u(5) f(0.10)<u(1)[1-f(0.99)+f(0.10)] ; L_{2}^{\prime} \succ L_{1}^{\prime}$ implies $u(5) f(0.10)>u(1) f(0.11)$, so that the simultaneous choices $L_{1} \succ L_{2}$ and $L_{2}^{\prime} \succ$ $L_{1}^{\prime}$ are explainable by RDU theory for any $f$ satisfying $: 1-f(099)>f(0.11)-$ $f(0.10)$, revealing that the same probability difference 0.01 is considered as more important in the neighborhood of certainty.

\subsubsection{Key axiom of RDU's axiomatization : comonotonic sure- thing principle}

The key axiom of the RDU model is the following :

Axiom 8 Comonotonic Sure-Thing Principle under risk ${ }^{8}$ :

Let $P$, and $Q$ be two lotteries of $\mathcal{L}$. $P=\left(x_{1}, p_{1} ; \ldots ; x_{k}, p_{k} ; \ldots ; x_{n}, p_{n}\right)$ and $Q=\left(y_{1}, p_{1} ; \ldots ; y_{k}, p_{k} ; \ldots ; y_{n}, p_{n}\right)$ be such that $x_{k_{0}}=y_{k_{0}} ;$ then $P \succeq Q$ implies $P^{\prime} \succeq Q^{\prime}$ for lotteries obtained from lotteries $P$ and $Q$ by merely replacing the $k_{0}^{\text {th }}$ common outcome $x_{i_{0}}$, by a common outcome $x k_{k_{0}}$ again in $k_{0}^{\text {th }}$ rank both in $P^{\prime}$ and $Q^{\prime}$.

This axiom from Chateauneuf (1999), is very similar to Green and Julien's ordinal independence axiom (1988), to Segal's irrelevance axiom (1987), and comonotonic independence in Chew and Wakker (1996)( see also ChewEpstein (1989), Quiggin (1989), Wakker, (1994) ; it is clearly much weaker than Savage's sure-thing principle that requires no restriction on $x_{i_{0}}$.

Remark 5 In the statement of this axiom, the common modification of the two lotteries do not change the order of the common outcomes in their respective distributions. The corresponding random variables canonically associated to the distributions ( $X$ and $Y$ taking respectively values $x_{k}$ and $y_{k}$ on sets $E_{k}$ with probability $p_{k}(k=1, . . n)$ stay comonotonic.

To capture the real meaning of this axiom, let us come back to Allais' experiment where subjects have to chose first between $L_{1}$ and $L_{2}$, then independently, between $L_{1}^{\prime}$ and $L_{2}^{\prime}$ where the 4 lotteries are indicated in the following table:

${ }^{8}$ The justification of the denomination of this axiom results from a natural interpretation of P, Q, P', Q' as probability distributions of pairwise comonotonic random variables. 


\begin{tabular}{ccccc} 
& & \multicolumn{3}{c}{ Pr Probabilities } \\
& & 0,01 & 0,89 & 0,1 \\
Lotteries & $L_{1}$ & $1 M$ & $1 M$ & $1 M$ \\
& $L_{2}$ & 0 & $1 M$ & $5 M$ \\
& $L_{1}^{\prime}$ & $1 M$ & 0 & $1 M$ \\
& $L_{2}^{\prime}$ & 0 & 0 & $5 M$
\end{tabular}

The common modification from $L_{1}$ to $L_{1}^{\prime}$ and from $L_{2}$ to $L_{2}^{\prime}$ does not preserve the rank of the common outcome in the both modified lotteries : more precisely, the common value $1 \mathrm{M}$ (with proba0.89) in $L_{1}$ and $L_{2}$ corresponds to an intermediate value whereas the common value $0 \mathrm{M}$ (with the same probability) corresponds to the smallest value. Thus, the two choices $L_{1}$ and $L_{2}^{\prime}$ do not contradict the previous Comonotonic Sure-Thing Principle.

A complete characterization of RDU model can be, for instance, obtained with the help of noncontradictory comonotonic trade-offs (see Wakker (1994)), or else with a comonotonic mixture independence axiom, an adaptation of mixture independence, which underlines the role played not only by comonotony, but also by the extreme outcomes (see Chateauneuf, 1998).

This attractive axiom is a central necessary axiom in the characterization of RDU, but, as in the case of CEU, even jointly with the standard axioms of weak order, continuity, monotony, this axiom fails to fully characterize RDU.

A complete characterization of RDU model can be, for instance, obtained with the help of noncontradictory comonotonic trade-offs (see Wakker (1994)), or else with a comonotonic mixture independence axiom, an adaptation of mixture independence, which underlines the role played not only by comonotony, but also by the extrema outcomes (see Chateauneuf, 1998).

More precisely, to characterize RDU model, Chateauneuf adds to the usual axioms of weak order, monotony, continuity and the Comonotonic Sure-Thing Principle under risk, the following axiom :

Axiom 9 Comonotonic Mixture Independence Axiom

$$
\begin{aligned}
& \text { For every } p \text { in }[0,1],(1) P_{1}=(1-p) \delta_{x_{1}}+p \delta_{a} \sim Q_{1}=(1-p) \delta_{y_{1}}+p \delta_{b} \text { and } \\
& \quad(2) P_{2}=(1-p) \delta_{x_{1}}+p \delta_{c} \sim Q_{2}=(1-p) \delta_{y_{1}}+p \delta_{d} \text { imply } \\
& \quad \text { (3) } \alpha P_{1}+(1-\alpha) P_{2} \sim \alpha Q_{1}+(1-\alpha) Q_{2}, \text { for every } \alpha \text { in }[0,1] \\
& \quad \text { For every } p \text { in }[0,1],(4) R_{1}=(1-p) \delta_{a}+p \delta_{z_{1}} \sim S_{1}=(1-p) \delta_{b}+p \delta_{t_{1}} \text { and } \\
& \text { (5) } R_{2}=(1-p) \delta_{c}+p \delta_{z_{1}} \sim S_{2}=(1-p) \delta_{d}+p \delta_{t_{1}} \text { imply } \\
& \quad \text { (6) } \alpha R_{1}+(1-\alpha) R_{2} \sim \alpha S_{1}+(1-\alpha) S_{2}, \text { for every } \alpha \text { in }[0,1] .
\end{aligned}
$$

This axiom underlines the role played not only by comonomonicity but also by the security factors $x_{1}$ and $y_{1}$ in (1) and (2)and potential factors ( $z_{1}$ and $t_{1}$ in (4) and (5) (see Jaffray's model (1988) and Cohen's model (1992) which are roughly summarized at the end of chapter 1$)$. 


\subsection{From CEU model to RDU model using first order stochastic dominance (Wakker)}

Let us first show that the RDU representation can be viewed as a Choquet integral .

\subsubsection{RDU representation is a Choquet integral}

In the RDU model, the function $f$ from $[0,1]$ to $[0,1]$ is increasing and satisfies $f(0)=$ 0 and $f(1)=1$. The corresponding "transformed" probability foP is thus a capacity and the RDU functional is a Choquet integral with respect to this capacity $v=f o P$. More precisely, $F(x))$

$V(Z)=\int_{C h} u(Z) d(f o P)=-\int_{-\infty}^{\infty} u(x) d f(P(Z>x))=-\int_{-\infty}^{\infty} u(x) d f(1-$

Remark 6 Let us notice by now, that if $f$ is a convex function, then $v=f_{o} P$ is a convex capacity (see e.g. Chateauneuf, (1991) or Denneberg, (1994)). Moreover, if $f$ is below the diagonal (i.e. satisfies $f(p) \leq p, \forall p \in[0,1]$ ), then it can be easily seen that core $(v) \neq \phi$.

\subsubsection{From CEU to RDU}

It has been recognized by several authors including Wakker (1990), Chateauneuf (1991), that RDU model under risk can be derived from CEU model under uncertainty by merely postulating the respect of first order stochastic dominance. We will use this approach, first to get Yaari's model from the simplified version of Schmeidler's model (section 2.2??), then to get RDU model from Choquet Expected Utility model.

Being under risk, we suppose that the objective probability $P$ is compatible with the preference relation on $(\mathbb{V}, \succeq)$. More precisely, we suppose :

Axiom 10 First order stochastic dominance :(Pour Alain A revoir)

$$
[A, B \in \mathcal{A}, P(A) \geq P(B)] \Rightarrow A \succeq B .
$$

Let us notice that this axiom is actually weaker than the first order stochastic dominance axiom but proves to be equivalent in this framework. This axiom implies also the neutrality axiom stated at the beginning of the section.

From simplified Schmeidler's model to Yaari's model Let us suppose that the preference relation on $(\mathbb{V}, \succeq)$ satisfies, moreover the usual axioms of non-trivial weak-order, continuity, monotonicity, the comonotonic independence axiom 2??,. The preference relation is then represented by a Choquet integral with respect to a capacity $v$ such that $A \succeq B$ implies $v(A) \geq v(B)$. The axioms imply then that $P(A) \geq P(B)$ imply $v(A) \geq v(B)$. 
This gives us an intuition of the result : There exists a unique transformed function $f$ : increasing function $f:[0,1] \rightarrow[0,1]$ satisfying $f(0)=0, f(1)=1)$ such that $v=f_{O P}$.

It can then be readily seen that the simplified Schmeidler's model reduces to Yaari's model under the assumption of first order stochastic dominance (Wakker, 1990, Chateauneuf, 1994).

From general CEU model to RDU model Let us suppose that the preference relation on $(\mathbb{V}, \succeq)$ satisfies all the axioms to get general CEU model characterized by $v$ and $u$ (see definition ??), then, $A \succeq B$ implies $v(A) \geq$ $v(B)$. If moreover, the objective measure $P$ on $S$ satisfies first order stochastic dominance, again, since $P(A) \geq P(B)$ imply $v(A) \geq v(B)$, there exists then a unique transformation function $f$ such that $v=f_{o P}$. We get the following result due to Wakker (1990) :

Let the preference relation on $(\mathbb{V}, \succeq)$ satisfies all the axioms to get general CEU, and let $P$ be a probability distribution on $\mathrm{S}$ satisfying first order stochastic dominance, then the preference relation on $(\mathbb{V}, \succeq)$ can be represented by the RDU model.

\subsection{Different notions of Risk Aversion and their characteriza- tion in the RDU model}

In the chapter 1 (decision under risk : the classical model), we already defined two notions of Risk Aversion (RA) : strong RA and weak RA. In the EU model, both notions have the same characterization: concavity of $u$. Let us recall these two notions here, before defining other ones.

The most natural way to define risk aversion is the following :

Definition 8 a $D M$ is weakly risk averse if he always prefers to any random variable $X$ the certainty of its expected value $E(X)$ (weakly risk-seeking if he always prefers any random variable $X$ the certainty to its expected value $E(X)$, risk-neutral if he is always indifferent between $X$ and $E(X)$ ).

An other possible way to define some type of risk aversion is to define it as aversion to some type of (mean preserving) increase in risk. All kinds of stochastic orders can then generate as many different kinds of risk aversion.

There exists, then, many different definitions of risk aversion but their different meanings have been hidden by the fact that, under expected utility theory, all are equivalent : they all reduce to the concavity of the utility function(see chapter 1 , section 4.3.).

Let us give some usual definitions of (mean preserving) increase in risk and their corresponding definitions of risk aversion: 


\subsubsection{Strong risk aversion}

$Y$ is a general mean preserving increase in risk (MPIR) of $X$ if $\int_{-\infty}^{t} F_{Y}(x) d x \geq$ $\int_{-\infty}^{t} F_{X}(x) d x$ for all $t \in \mathbb{R}$ and $\int_{-\infty}^{+\infty} F_{Y}(x) d x=\int_{-\infty}^{+\infty} F_{X}(x) d x$. This usual concept of (mean preserving) increasing risk is classically used in economics, since Rothschild and Stiglitz (1970) and we define the corresponding notion of strong risk aversion :

Definition 9 A DM is then strongly risk averse if he is averse to any general (Mean Preserving) increase in risk, i.e. for any $X$ and $Y$ in $\mathbb{V}$ such that $Y$ is a MPIR of $X$, he prefers $X$ to $Y$ (strongly risk seeking if he prefers $Y$ to $X$, risk neutral if he is indifferent).

\subsubsection{Monotone risk aversion}

Quiggin (1992) brought to light that strong risk aversion may be a too strong concept, and introduced a new notion, monotone (mean-preserving) increase in risk, defined in terms of comonotonic random variables instead of a general mean-preserving increasing risk :

$Y$ is a (mean preserving) monotone increase in risk (MPMIR) of $X$ if and only if ${ }^{9} Y=X+Z$, where $Z$ is such that $E(Z)=0$ and $X$ and $Z$ are comonotonic.

Before giving an important property of this notion, let us recall that $F^{-1}(p)=\inf \{z \in \mathbb{R} \mid F(z) \geq p\}$, and then we can interpret $F^{-1}(p)$ as the highest gain among the least favorable $p \%$ of the outcomes.

Property : Lansberger and Meilijson (1994b) have proved that for two random variables with equal mean this notion is equivalent to the statistical notion of "dispersion" introduced by Bickel and Lehmann $(1976,1979): Y$ is more dispersed than $X$ if

$F_{Y}^{-1}(q)-F_{Y}^{-1}(p) \geq F_{X}^{-1}(q)-F_{X}^{-1}(p)$, where $F^{-1}$ is defined from $(0,1]$ into $\mathbb{R}$ by $F^{-1}(p)=\inf \{z \in \mathbb{R} \mid F(z) \geq p\}$, for all $0<p<q<1$.

Thus, if $Y$ is MPMIR of $X$, all the interquantile intervals are shorter for $X$ than for $Y$. Let us then define the corresponding notion of Monotone Risk Aversion.

Definition 10 A DM is monotone risk averse if he is averse to any monotone increase in risk i.e. for every pair $(X, Y)$ where $Y$ is $M I R$ of $X$, he always prefers $X$ to $Y$ (monotone risk-seeking if he always prefers $Y$ to $X$, risk-neutral if he is always indifferent between $X$ and $Y$.

This notion of monotone risk aversion ${ }^{10}$ is particularly fitted to RDU theory where comonotony plays a fundamental part at the axiomatic level.

\footnotetext{
${ }^{9}=$ means equality of probability distributions.

${ }^{10} \mathrm{~A}$ pplications of this notion of risk aversion can be found e.g. in Cohen (1995).
} 


\subsubsection{Left monotone risk aversion}

The order induced by monotone increasing risk is a very partial order since it can order very few pairs of random variables. The following notion compares more pairs and this notion of increasing risk is asymmetric in the sense that it treats differently downside and upside risks. This notion will prove to be particularly fitted with deductible insurance (see Vergnaud, 1997).

The following definition is due to Jewitt ${ }^{11}$ (1989) under the name of Locationindependent Risk (see also Lansberger and Meilijson (1994a). The motivation of Jewitt was to find a notion of increase in risk that models coherent behavior in a context of partial insurance ${ }^{12}$.

$Y$ is said to be a left monotone mean preserving increase in risk (LIR) of $X$ if $\int_{-\infty}^{F_{Y}^{-1}(p)} F_{Y}(x) d x \geq \int_{-\infty}^{F_{X}^{-1}(p)} F_{X}(x) d x$ for all $p \in(0,1)$.

Again, we define the corresponding notion of left monotone risk aversion :

Definition 11 A DM is left monotone risk averse (respectively, left monotone risk seeking) if he is averse to any left monotone increase in risk, i.e. for any $X$ and $Y$ in $\mathbb{V}$ such that $Y$ is a left monotone MPIR of $X$, the DM prefers $X$ to $Y$ (respectively, $Y$ to $X$ ).

Remark 7 It can be readily seen that Strong risk aversion $\Rightarrow$ Left motone risk aversion $\Rightarrow$ Monotone risk aversion $\Rightarrow$ Weak risk aversion. The reverse implications are not true, in general. However, in the EU model, all these notions are equivallent and are reduced to the concavity of $u$.

\subsubsection{Characterization of different notions of risk aversion in the RDU model}

Contrary to EU model, in the RDU model, each of the different notions of aversion to risk has a specific characterization ${ }^{13}$. Gathering several results in different papers, we get the following results.

Let a RDU decision maker be characterized by two differentiable functions $u$ and $f$ :

1. A RDU Decision Maker is then strongly risk averse (respectively strongly risk-seeking) if and only if the utility function $u$ is concave and the transformation function $f$ is convex (respectively $u$ convex and $f$ concave) (see Chew, Karni and Safra, 1987). ${ }^{14}$

\footnotetext{
${ }^{11}$ In Jewitt, the notion is given for $X$ and $Y$ with possibly different means.

${ }^{12}$ See also Lansberger and Meilijson (1994a) on this subject.

${ }^{13}$ Machina $(1982 \mathrm{a}, 1982 \mathrm{~b})$ was the first to notice that the equivalence between different notions of risk aversion in the EU model does not carry over to generalized models.

${ }^{14} \mathrm{f}$ convex implies that the capacity $f o P$ is convex.
} 
2. A RDU Decision Maker is left monotone risk averse if and only if his transformation function $f$ is star-shaped at 1 from above ${ }^{15}$ and his utility function $u$ is concave (see Chateauneuf, Cohen and Meilijson, 2004).

3. A RDU Decision Maker is left monotone risk-seeking if and only if the transformation function $f$ is star-shaped at 1 from below ${ }^{16}$ and the utility function $u$ is convex (see Chateauneuf, Cohen and Meilijson, 2004).

4. The characterization of monotone risk aversion is based on two following indices : $P_{f}=\inf _{0<v<1}\left[\frac{1-f(v)}{f(v)} / \frac{1-v}{v}\right]$, called index of pessimism, which is $\geq 1$ as soon as $f(p) \leq p$, and $G_{u}=\sup _{y \leq x} u^{\prime}(x) / u^{\prime}(y)$, called index of nonconcavity $^{17}$ (or greediness), which always satisfies $G_{u} \geq 1$, and where the value 1 corresponds exclusively to concavity.

A RDU Decision Maker with probability transformation function $f$ and differentiable utility $u$ is monotone risk averse if and only if his index of pessimism is greater than his index of non-concavity, i.e. $P_{f} \geq G_{u}$ (see Chateauneuf, Cohen and Meilijson, 2005). The most significant feature of this result is that a DM does not need to have a concave utility function $u$ to be monotone risk averse.

5. The characterization of monotone risk-seeking is based on two following indices : $O_{f}=\inf _{0<v<1}\left[\frac{f(v)}{1-f(v)} / \frac{v}{1-v}\right]$ called index of optimism, which is $\geq 1$ as soon as $f(p)>p$, and $T_{u}=\sup _{x<y} \frac{u^{\prime}(x)}{u^{\prime}(y)}$, called index of nonconvexity, which always satisfies $T_{u} \geq 1$ and the value 1 corresponds exclusively to convexity.

A RDEU DM with probability perception function $f$ and utility function $u$ is monotone risk-seeking if and only if the DM's index of optimism exceeds the DM's index of non-convexity: $O_{f} \geq T_{u}$.

6. For a weak risk averse RDU decision maker, there is no known characterization but sufficient conditions, not implying concavity of $u$ (see Chateauneuf and Cohen, 1994).

The interesting point of all these results is that RDU models not only allow to separate transformation of probability from valuation of outcomes but moreover explain much diversified behaviors as, for instance, to be weakly risk-seeking with a diminishing marginal utility of wealth or to dislike risk (to

\footnotetext{
${ }^{15} \mathrm{~A}$ transformation function $f:[0,1]$ to $[0,1]$ is star-shaped at 1 from above if for any $x$ of $\mathbb{R}, x<1, \frac{1-f(x)}{1-x}$ is increasing.

${ }^{16} \mathrm{~A}$ transformation function $f:[0,1]$ to $[0,1]$ is is star-shaped at 1 from below if, for any $x$ of $\mathbb{R}, x<1, \frac{1-f(x)}{1-x}$ is decreasing.

${ }^{17}$ if $u$ is not differentiable (see Chateauneuf, Cohen and Meilijson, 1997), in which case $G_{u}$ becomes more complex: $G_{u}=\sup _{x_{1}<x_{2} \leq x_{3}<x_{4}} \frac{u\left(x_{4}\right)-u\left(x_{3}\right)}{x_{4}-x_{3}} / \frac{u\left(x_{2}\right)-u\left(x_{1}\right)}{x_{2}-x_{1}}$
} 
be weak risk averse) but to accept sometimes a (mean preserving) increase in risk (not to be a strong risk averse).

All the cardinal extensions of the EU model proposed in this chapter allow for a better representation of real behavior under uncertainty.

Before ending the chapter, let us note that there exist other cardinal generalizations of the subjective EU model allowing for further explanations of Ellsberg paradox. Let us roughly mention some of them for interested readers : P. Ghirardato F. Macceroni, M. Marinacci, (2005), P. Klibanoff, M. Marinacci and S. Mukerji, (2005), F., Macceroni, M. Marinacci and A. Rustichini (2006), A. Chateauneuf, S. Grant and J. Eichberger (2007), (T. Gajdos, T. Hayashi, J.-M. Tallon and J.-C. Vergnaud (2008), Ghirardato and Marinacci $?$

We will see, in the following chapter, "ordinal " extentions of the EU model.

\section{References}

[1] Allais, M.,. "Le comportement de l'homme rationnel devant le risque: critique des postulats et axiomes de l'école américaine", Econometrica, 21, 1953, p. 503-546.

[2] Allais, M." The general theory of random choices in relation to the invariant cardinal utility function and the specific probability function", in Risk, Decision and Rationality, B. R. Munier (Ed.), Reidel: Dordrecht, 1988, p. 233-289.

[3] Anscombe F.J., and Aumann, R.J." A definition of subjective probability", The annals of mathematical statistics, 34, p 199-205, 1963.

[4] Bickel, P. J. and E. L. Lehmann. "Descriptive statistics for nonparametric models, III. Dispersion", Annals of Statistics, 4, p. 1139-1158, 1976.

[5] Bickel, P. J. and E. L. Lehmann. "Descriptive statistics for nonparametric models, IV. Spread",in Contributions to Statistics, Jureckova (Ed.), Reidel, 1979.

[6] Chateauneuf, A. "On the use of capacities in modeling uncertainty aversion and risk aversion", Journal of Mathematical Economics, 20, p 343369, 1991.

[7] Chateauneuf, A. "Modeling attitudes towards uncertainty and risk through the use of Choquet integral", Annals of Operations Research, 52, p. 3-20, 1994.

[8] Chateauneuf, A. "Comonotonicity axioms and RDU theory for arbitrary consequences", Journal of Mathematical Economics, 32, 21-45, 1999. 
[9] Chateauneuf, A. and M. D. Cohen. "Risk-seeking with diminishing marginal utility in a non-expected utility model", Journal of Risk and Uncertainty, 9, p. 77-91, 1994.

[10] Chateauneuf, A., M. D. Cohen and R. Kast. "A review of some results related to comonotony", cahiers d'ecomath, $\mathrm{n}^{0}$ 97.32, 1997.

[11] Chateauneuf, A., M. D. Cohen and I. Meilijson. "More pessimism than greediness: A characterization of monotone risk aversion in the Rank Dependent Expected Utility model", Economic Theory, 25, 3, 649-668, 2005.

[12] Chateauneuf, A., M. D. Cohen and I. Meilijson. "Four notions of meanpreserving increase in risk, risk attitudes and applications to the RankDependent Expected Utility Model", Journal of Mathematical Economics, 40, 547-571, 2004.

[13] Chateauneuf A. and J.M.Tallon. "Diversification, convex preferences and non-empty core in the Choquet expected utility model", Economic Theory, 19, 509-523, 2002.

[14] Chew, S and L. Epstein. "A unifying approach to axiomatic non-expected utility theory, Journal of Economic Theory, 49, p207-240, 1989.

[15] Chew, S., L. Epstein and P. Wakker. "A unifying approach to axiomatic non-expected utility theory: correction and comment", Journal of Economic Theory, 59, 183-188, 1993.

[16] Chew, S., E. Karni and Z. Safra. "Risk aversion in the theory of expected utility with Rank Dependent preferences", Journal of Economic Theory, 42, p. 370-381, 1987.

[17] Chew, S. and P.Wakker. "The comonotonic sure-thing principle", Journal of risk and uncertainty, 12, p. 5-27, 1996.

[18] Choquet, G. "Théorie des capacités", Ann. Institut Fourier (Grenoble), V, p. 131-295,1953.

[19] Cohen, M.D. "Security level, potential level, expected utility : a threecriteria decision model under risk", Theory and Decision, Vol33, 2, 1-34, 1992.

[20] Cohen, M. D. "Risk aversion concepts in expected and non-expected utility models", The Geneva Papers on Risk and Insurance theory, 20, p. 73-91, 1995.

[21] Dempster, A.P. "Upper and lower probabilities induced by a multivalued mapping", Annals of Mathematical Statistics, 38, p325-339, 1967. 
[22] Denneberg, D. "Non-additive measure and integral", Kluwer Academic Publishers, 1994.

[23] Ellsberg, D. "Risk, ambiguity and the Savage axioms", Quartely Journal of Economics, 75, p643-669, 1961.

[24] Gayant, J.P." Arguments graphiques simples pour comprendre la spécification du modèle d'espérance d'utilité et l'intégrale de Choquet", Actualité économique, 74, 183-195, 1998.

[25] Gilboa, I. "Expected utility with purely subjective non-additive probabilities" Journal of Mathematical Economics, 16, p. 65-88, 1987.

[26] Gilboa, I. (Ed). "Uncertainty in Economic Theory, Essays in honor of David Schmeidler's 65th birthday", Routledge, 2004.

[27] Gilboa I. and D. Schmeidler." Maxmin expected utility with a non-unique prior", Journal of Mathematical Economics, 18, 141-153, 1989.

[28] Green, J. and B. Jullien. "Ordinal independence in non-linear utility theory, Journal of risk and uncertainty, 1, p355-387, 1988.

[29] Jaffray, JY. "Choice under risk and the security factor. An axiomatic model". Theory and Decision, Vol 24, 2, 1988.

[30] Jaffray, JY. "Généralisation du critère de l'utilité espérée aux choix dans l'incertain régulier", Recherche Opérationnelle, 23, p. 237-267, 1989 a.

[31] Jaffray, JY. " Linear utility for belief functions", Operations Recherch Letters, 8, p. 107-112, 1989b.

[32] Jaffray, J.Y. and F. Philippe." On the existence of subjective upper and lower probabilities", Mathematics of Operations Research, 22, p 165-185, 1997.

[33] Jewitt, I. "Choosing between risky prospects : the characterization of comparative statics results, and location independent risk", Management Science, 35, p. 60-70, 1989.

[34] Kahneman, D. and A. Tversky. "Prospect Theory : an analysis of decision under risk", Econometrica, 47, p263-291, 1979.

[35] Peter Klibanoff \& Massimo Marinacci \& Sujoy Mukerji, 2005. "A Smooth Model of Decision Making under Ambiguity," Econometrica, Econometric Society, vol. 73(6), pages 1849-1892,

[36] Landsberger, M. and I. Meilijson. "The generating process and an extension of Jewitt's location independent risk concept", Management Science, 40, p. 662-669, 1994a. 
[37] Landsberger, M. and I. Meilijson. "Comonotone allocations, BickelLehmann dispersion and the Arrow-Pratt measure of risk aversion", $A n$ nals of Operations Research, 52, p. 97-106, 1994b.

[38] Econometrica Volume 74 Issue 6, Pages 1447 - 1498Ambiguity Aversion, Robustness, and the Variational Representation of Preferences

[39] Machina, M. "Expected utility analysis without the independence axiom", Econometrica, 50, p. 277-323, 1982a.

[40] Machina, M. "A stronger characterization of declining risk aversion", Econometrica, 50, $\mathrm{n}^{0} 4$, p 1069-1079, 1982b.

[41] Machina, M. and D. Schmeidler." A more robust definition of subjective probability ", Econometrica, 60, p745-780, 1992.

[42] Quiggin, J. "A theory of anticipated utility",Journal of Economic Behavior and Organisation, 3, p. 323-343, 1982.

[43] Quiggin, J. "Increasing risk: another definition", In Progress in Decision, Utility and Risk Theory, A. Chikan (Ed.), Dordrecht: Kluwer, 1992.

[44] Rothschild, M. and J. Stiglitz." Increasing Risk: I. A definition", Journal of Economic Theory, 2, p. 225-243, 1970.

[45] Savage, L. "The foundations of statistics. New York. Wiley, (Second edition 1972, Dover), 1954.

[46] Schmeidler, D. " Integral representation without additivity", Proceedings of the American Mathematical Society, 97, p. 255-261, 1986.

[47] Schmeidler, D. "Subjective probability and expected utility without additivity" Econometrica, 57, p. 517-587, 1989. (First version: Subjective expected utility without additivity, Forder Institute Working Paper (1982)).

[48] Segal, U. "Anticipated utility: a measure representation approach", Annals of Operations Research, 19, p. 359-374, 1987.

[49] Segal, U. "The measure representation : A correction", Journal of Risk and Uncertainty, 6, p. 99-107, 1993.

[50] Shafer, G. " A mathematical theory of evidence", Princeton University Press, Princeton, NJ, 1976.

[51] Tversky, Amos and Kahneman, Daniel. "Advances in Prospect Theory: Cumulative Representation of Uncertainty", Journal of Risk and Uncertainty, Volume 5, 4, 297-323, 1992. 
[52] Vergnaud, J.C. "Analysis of risk in a non-expected utility framework and applications to the optimality of the deductible", Revue Finance, 18, 1, p155-167, 1997.

[53] von Neumann, J. and Morgenstern, O. "Theory of games and economic behavior", Princeton University Press, Princeton, N.J., 1947.

[54] Wakker, P. "Continuous subjective expected utility with non-additive probabilities", Journal of Mathematical Economics, 1-27, 1989.

[55] Wakker, P. "Under Stochastic Dominance Choquet Expected Utility and Anticipated Utility are identical", Theory and Decision, 29, 119-132, 1990.

[56] Wakker, P. "Separating marginal utility and risk aversion", Theory and Decision, 36, p. 1-44, 1994.

[57] Yaari, M. "The dual theory of choice under risk", Econometrica, 55, p. 95-115, 1987.

[58] Certainty Independence and the Separation of Utility and Beliefs, with P. Ghirardato, Fabio Maccheroni and Massimo Marinacci. Journal of Economic Theory, 120: 129-136, January 2005.

[59] Attitude toward imprecise information (with T. Hayashi, J.-M. Tallon and J.-C. Vergnaud), Journal of Economic Theory, 140, 27-65 (2008).

[60] GHTVAttitude toward imprecise information

Available online 6 October 2007

Journal of Economic Theory

KMM "A smooth model of decision making under ambiguity" (, Econometrica 73(6) (November, 2005) pp. 1849-1892.

MMR (variational) Fabio Maccheroni \& Massimo Marinacci \& Aldo Rustichini, 2006. "Ambiguity Aversion, Robustness, and the Variational Representation of Preferences," Econometrica, Econometric Society, vol. 74(6), pages 1447-1498, November.

NEO Grant, Simon with A. Chateauneuf and J. Eichberger "Choice Under Uncertainty with the Best and Worst in Mind: Neo-Additive Capacities." Journal of Economic Theory, 137 (2007): 538-567.

Faro et chateauneufAmbiguity through Confidence Functions

by Alain Chateauneuf and José Heleno Faro

GM (bi-separable) Risk, Ambigity and the Separation of Utility and Beliefs 